\title{
ON MEASURABILITY, POINTWISE CONVERGENCE AND COMPACTNESS ${ }^{1}$
}

\author{
BY A. IONESCU TULCEA
}

The starting point of this investigation is the beautiful generalization of Egorov's theorem given by P. A. Meyer in Séminaire de Probabilités. $\mathrm{V}$, (Strasbourg). The material is divided as follows:

$\S 1$. Setting and terminology.

§. The Generalized Egorov Theorem.

$\S 3$. An application to vector-valued mappings.

$\S 4$. The $\langle\langle$ separation property $\rangle\rangle$ and the notion of lifting.

Proofs of most of the results contained in this paper can be found in $[5],[6],[7],[8]$.

1. Setting and terminology. Throughout this article $(E, \mathscr{E}, \mu)$ will be a fixed probability space. We denote by $\mathscr{L}=\mathscr{L}(E, \mathscr{E}, \mu)$ the algebra of all $f: E \rightarrow R$ which are $\mathscr{E}$-measurable.

For $f \in \mathscr{L}, g \in \mathscr{L}$ we write

and

$$
f \equiv g \quad \text { if } f(t)=g(t) \quad \mu \text {-almost surely, }
$$

$$
f=g \quad \text { if } f(t)=g(t) \text { for all } t \in E .
$$

For $f \in \mathscr{L}$, we denote by $\tilde{f}$ the equivalence class of $f$ with respect to the equivalence relation " $\equiv$ " defined above.

We denote by $\mathscr{L}^{\infty}=\mathscr{L}^{\infty}(E, \mathscr{E}, \mu)$ the algebra of all bounded $f \in \mathscr{L}$.

For a set $B \in \mathscr{E}$ we denote by $1_{B}$ the indicator function of $B\left(\right.$ i.e. $1_{B}(t)=1$ for $t \in B$ and $1_{B}(t)=0$ for $\left.t \in E-B\right)$.

We say that a set $A \in \mathscr{E}$ carries $\mu$ if $\mu(E-A)=0$.

2. The Generalized Egorov Theorem. We may now state P. A. Meyer's generalization of Egorov's theorem (see [8, p. 199]) as follows:

Theorem 1 (Generalized Egorov TheORem). Let $H \subset \mathscr{L}$ be compact and metrizable for the topology of pointwise convergence on $E$. There exists

Invited address presented at the Evanston Meeting of the American Mathematical Society on April 28, 1973; received by the editors May 23, 1973.

AMS (MOS) subject classifications (1970). Primary 28A20, 46G15.

${ }^{1}$ Research supported by the U.S. Army Research Office (Durham) under contract No. DAHCO4 68 C005. 
then a sequence $\left(A_{n}\right)$ of disjoint subsets of $E$ such that $A_{n} \in \mathscr{E}$ for each $n$, and $\bigcup_{n} A_{n}$ carries $\mu$, with the following property:

If $\left(h_{j}\right)$ is any sequence of elements of $H$, converging pointwise on $E$, say to $h$, then for each $n,\left.h_{j}\right|_{A_{n}}$ converges uniformly to $\left.h\right|_{A_{n}}$.

REMARK 1. It should be stressed that the decomposition $\left(A_{n}\right)$ is independent of the particular sequence $\left(h_{j}\right)$ in $H$.

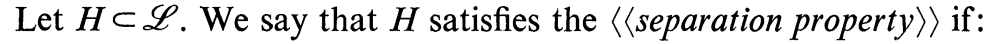

$$
h_{1} \in H, h_{2} \in H, h_{1} \neq h_{2} \Rightarrow \tilde{h}_{1} \neq \tilde{h}_{2} \text {. }
$$

The relevant comment on the Generalized Egorov Theorem and the $\langle\langle$ separation property $\rangle\rangle$ is formulated in Theorem 2 below (see [5]; see also the notion of $\langle\langle$ partitionable function $\rangle\rangle$ introduced by M. Sion [10, p. 590]):

THEOREM 2. Let $H \subset \mathscr{L}$ be compact metrizable for the topology of pointwise convergence on $E$. Then there is a set $E_{0} \in \mathscr{E}$ carrying $\mu$, with the following properties:

(1) For each $\varepsilon>0$ there is a partition $\left(A_{n}^{\varepsilon}\right)$ of $E_{0}$ with $A_{n}^{\varepsilon} \in \mathscr{E}$ and $\mu\left(A_{n}^{\varepsilon}\right)>0$ for each $n$, such that:

$$
s \in A_{n}^{\varepsilon}, t \in A_{n}^{\varepsilon}, h \in H \Rightarrow|h(s)-h(t)| \leqq \varepsilon .
$$

(2) $\left.H\right|_{E_{0}}$ satisfies the 〈〈separation property $\left.\rangle\right\rangle$.

We next make some remarks concerning the topology of pointwise convergence on a set of measurable functions:

REMARK 2. Let $H=\left\{h_{1}, h_{2}, \cdots, h_{n}, \cdots\right\}$ where each $h_{n}=1_{B_{n}}$, with $B_{n} \in \mathscr{E}$. We may identify $H$ with a subset of the compact space $\{0,1\}^{E}$; the topology of pointwise convergence is then simply the product space topology. Various pathologies may occur:

(2.1) One may construct a sequence $\left(h_{n}\right)$ such that every cluster value of this sequence is non-measurable (see for instance [1, Chapter IV (1952), p. 199, Exercise 4]).

(2.2) One may find $H=\left\{h_{1}, h_{2}, \cdots\right\}$ such that $\bar{H}=\{0,1\}^{E}$.

Therefore the following questions are of interest:

Question 1. Let $H \subset \mathscr{L}$ be countable. Under what conditions is $\bar{H}$ compact metrizable (for the topology of pointwise convergence on $E$ )?

Question 2. Let $H \subset \mathscr{L}$ be compact. Under what conditions is $H$ metrizable (for the topology of pointwise convergence on $E$, of course)?

We shall begin with Question 2.

The following is a partial answer to Question 2, which however suffices for practical purposes (in Theorem 3 below we consider of course $H$ endowed with the topology of pointwise convergence on $E$ ): 
Theorem 3 (Metrization Criterion). Let $H \subset \mathscr{L}$ be a set with the following properties:

(i) $H$ is compact.

(ii) $H$ is convex.

(iii) $H$ satisfies the $\langle\langle$ separation property $\rangle\rangle$.

Then $H$ is metrizable.

To prove Theorem 3 one shows that, under our assumptions, the topology of pointwise convergence and the topology of convergence in probability coincide (see [6]). One may use in the proof the following remarkable theorem due to Komlós (see [7] or [2]):

THEOREM 4 (Kolmós). Let $\left(f_{n}\right)$ be a sequence of elements of $\mathscr{L}^{1}(E, \mathscr{E}, \mu)$ with $\sup _{n}\left\|f_{n}\right\|_{1}<\infty$. Then one can find a subsequence $\left(f_{n_{k}}\right)$ and an element $f \in \mathscr{L}^{1}(E, \mathscr{E}, \mu)$ such that $\left(f_{n_{k}}\right)$, as well as any further subsequence extracted from $\left(f_{n_{k}}\right)$, converges Cesàro to $f, \mu$-almost surely.

3. An application to vector-valued mappings. Let $X$ be a Banach space, $X^{\prime}$ its dual. We denote the duality by $\left\langle x^{\prime}, x\right\rangle, x \in X, x^{\prime} \in X^{\prime}$. Let now $g: E \rightarrow X$. For $x^{\prime} \in X^{\prime}$ we denote by $\left\langle x^{\prime}, g\right\rangle$ the mapping $t \rightarrow\left\langle x^{\prime}, g(t)\right\rangle$ of $E$ into $R$.

We recall that $g: E \rightarrow X$ is called weakly measurable if the real-valued mapping $\left\langle x^{\prime}, g\right\rangle$ is $\mathscr{E}$-measurable for each $x^{\prime} \in X^{\prime}$. We recall also that $g: E \rightarrow X$ is called strongly (Bochner) measurable if there is a sequence $\left(s_{n}\right)$ of simple functions such that $\lim _{n} s_{n}(t)=g(t), \mu$-almost surely.

We may now state the following theorem (see [6]):

THeOREM 5 (WEAK VERSUS STRONG MEASURABILITy). Let $g: E \rightarrow X$ be weakly measurable. We have:

(1) Suppose that the relations $x^{\prime} \in X^{\prime}, y^{\prime} \in X^{\prime}$ and $\left\langle x^{\prime}, g\right\rangle \neq\left\langle y^{\prime}, g\right\rangle$ imply $\left\langle x^{\prime}, g\right\rangle \not \equiv\left\langle y^{\prime}, g\right\rangle$. Then $g$ is strongly measurable.

(2) Conversely, if $g: E \rightarrow X$ is strongly measurable, there is a set $\cdot E_{0} \in \mathscr{E}$ carrying $\mu$ such that the relations $x^{\prime} \in X^{\prime}, y^{\prime} \in X^{\prime}$ and $\left.\left\langle x^{\prime}, g\right\rangle\right|_{E_{0}} \neq\left.\left\langle y^{\prime}, g\right\rangle\right|_{E_{0}}$ imply $\left\langle x^{\prime}, g\right\rangle \not \equiv\left\langle y^{\prime}, g\right\rangle$.

It appears, therefore, that the $\langle\langle$ separation property $\rangle\rangle$ really makes the difference between weak measurability and strong measurability.

4. The $\langle\langle$ separation property $\rangle\rangle$ and the notion of lifting. The most convenient way to obtain the $\langle\langle$ separation property $\rangle\rangle$, at least for sets of bounded measurable functions, is by applying the notion of lifting:

We recall that a mapping $\rho: \mathscr{L}^{\infty} \rightarrow \mathscr{L}^{\infty}$ is called a lifting of $\mathscr{L}^{\infty}$ if it 
satisfies the following conditions:

(I) $\rho(f) \equiv f$;

(II) $f \equiv g$ implies $\rho(f)=\rho(g)$;

(III) $\rho(1)=1$;

(IV) $\rho(a f+b g)=a \rho(f)+b \rho(g)$;

(V) $\rho(f g)=\rho(f) \rho(g)$.

Without going into the history of the subject, it suffices to recall that if $(E, \mathscr{E}, \mu)$ is a complete probability space, then a lifting of $\mathscr{L}^{\infty}$ always exists (see for instance [4]).

Henceforth we assume that $(E, \mathscr{E}, \mu)$ is a complete probability space.

There is an equivalent way of defining the notion of lifting if one prefers to work with sets rather than functions. For each $A \in \mathscr{E}, \rho\left(1_{A}\right)$ is again an indicator function (by axiom (V)); we write

$$
\rho\left(1_{A}\right)=1_{\rho(A)} \text {. }
$$

The mapping $\rho: \mathscr{E} \rightarrow \mathscr{E}$ obtained in this manner satisfies the conditions:

$\left(\mathrm{I}^{\prime}\right) \rho(A) \equiv A$;

(II') $A \equiv B$ implies $\rho(A)=\rho(B)$;

(III') $\rho(E)=E, \rho(\varnothing)=\varnothing$;

$\left(\mathrm{IV}^{\prime}\right) \rho(A \cup B)=\rho(A) \cup \rho(B)$;

$\left(\mathrm{V}^{\prime}\right) \rho(A \cap B)=\rho(A) \cap \rho(B)$.

The mapping $\rho: \mathscr{E} \rightarrow \mathscr{E}$ satisfying axioms $\left(\mathrm{I}^{\prime}\right)-\left(\mathrm{V}^{\prime}\right)$ is called a lifting of $\mathscr{E}$. Since this cannot lead to confusion, we shall use the same notation for the lifting of $\mathscr{L}^{\infty}$ and the corresponding lifting of $\mathscr{E}$.

Lifting topology. Let now $\rho$ be a fixed lifting of $\mathscr{L}^{\infty}=\mathscr{L}^{\infty}(E, \mathscr{E}, \mu)$. Corresponding to the lifting $\rho$ we may introduce a topology $\mathscr{T}_{\rho}$ on the space $E$ as follows:

$$
\mathscr{T}_{\rho}=\{\rho(A)-N \mid A \in \mathscr{E}, N \in \mathscr{E}, \mu(N)=0\} .
$$

The topology $\mathscr{T}_{\rho}$ turns out to have the following properties (see [4, p. 59]):

(1) $\mathscr{T}_{\rho}$ is extremally disconnected.

(2) $C_{R}^{b}\left(E, \mathscr{T}_{\rho}\right)=\left\{\rho(g) \mid g \in \mathscr{L}^{\infty}\right\}$.

We may now give an answer to Question 1 raised in $\$ 2$.

We shall only consider the case of a bounded set $H \subset \mathscr{L}^{\infty}$. We have the following analogue of Arzela-Ascoli's theorem (see [5], [6]):

THEOREM 6. Let $H \subset \mathscr{L}^{\infty}$ be a bounded set. We have:

(1) Suppose that $H$ is compact metrizable for the topology of pointwise convergence on $E$. There is then a set $E_{0} \in \mathscr{E}$ carrying $\mu$, such that $\left.H\right|_{E_{0}} \subset$ $\left.C_{R}^{b}\left(E, \mathscr{T}_{\rho}\right)\right|_{E_{0}}$ and $\left.H\right|_{E_{0}}$ is equicontinuous on (the $\mathscr{T}_{\rho}$-open set) $E_{0}$ with respect to $\mathscr{T}_{\rho}$.

(2) Conversely, suppose that $H \subset C_{R}^{b}\left(E, \mathscr{T}_{\rho}\right)$ and that $H$ is equicontinuous 
with respect to $\mathscr{T}_{\rho}$. Then $\bar{H}$ (closure of $H$ for the topology of pointwise convergence on $E$ ) is compact metrizable.

Another application. Let $(E, \mathscr{E}, \mu)$ be a complete probability space and $Z$ a completely regular topological space.

We recall the definition of the abstract space $\mathscr{L}_{Z}^{\infty}=\mathscr{L}_{Z}^{\infty}(E, \mathscr{E}, \mu)$. A mapping $f: E \rightarrow Z$ belongs to $\mathscr{L}_{Z}^{\infty}$ if:

(i) $f(E) \subset Z$ is relatively compact;

(ii) $f: E \rightarrow Z$ is weakly measurable, that is, $h \circ f$ is $\mathscr{E}$-measurable, for each $h \in C_{R}(Z)$.

It is clear that if $Z=R$, then $\mathscr{L}_{R}^{\infty}=\mathscr{L}^{\infty}$.

Let now $\rho$ be a lifting of $\mathscr{L}^{\infty}$. Starting with $\rho$ one may define an $\langle\langle$ abstract lifting $\rangle\rangle$ of the abstract space $\mathscr{L}_{Z}^{\infty}$ as follows: For $f \in \mathscr{L}_{Z}^{\infty}$ we set

$$
h \circ \rho_{Z}(f)=\rho(h \circ f), \text { for all } h \in C_{R}(Z) \text {. }
$$

The above "weak invariance formula" uniquely determines the abstract lifting $\rho_{Z}$ associated with $\rho$ (see [4, pp. 52-53]). Since there can be no confusion, we shall denote this abstract lifting by $\rho$ again.

This notion of abstract lifting has many advantages: let us mention in passing that it permits to give a very simple and rapid proof of Doob's classical theorem concerning the "existence of a separable modification" of a stochastic process (see [3] or [4]).

Let us now consider again a Banach space $X$ and let us return to weakly measurable versus strongly measurable mappings. ${ }^{2}$

Consider $\left(X, \sigma\left(X, X^{\prime}\right)\right)$ and correspondingly the abstract space $\mathscr{L}_{\left(X, \sigma\left(X, X^{\prime}\right)\right)}^{\infty}$.

We note that $f \in \mathscr{L}_{\left(X, \sigma\left(X, X^{\prime}\right)\right)}^{\infty}$ if

(i) $f(E) \subset X$ is $\sigma\left(X, X^{\prime}\right)$-relatively compact.

(ii) $\left\langle x^{\prime}, f\right\rangle$ is $\mathscr{E}$-measurable, for each $x^{\prime} \in X^{\prime}$.

We then have (see [5]):

TheOrem 7. Let $f \in \mathscr{L}_{\left(X, \sigma\left(X, X^{\prime}\right)\right)}^{\infty}$ and let $g=\rho(f)$. Then

$$
\rho\left(\left\langle x^{\prime}, g\right\rangle\right)=\left\langle x^{\prime}, g\right\rangle, \text { for each } x^{\prime} \in X^{\prime}
$$

and hence $g$ is strongly measurable.

It is enough to note that since $g$ satisfies the above invariance property, $g$ has the separation property required in Theorem 5, and therefore $g$ is strongly measurable.

\footnotetext{
${ }^{2}$ Below we denote by $\sigma\left(X, X^{\prime}\right)$ the $X^{\prime}$-topology on $X$, that is the weakest topology on $X$ making every linear functional $x^{\prime} \in X^{\prime}$ continuous.
} 
REMARK 3. The fact that if $f: E \rightarrow X$ is a weakly measurable mapping taking values in a $\sigma\left(X, X^{\prime}\right)$-compact set, then its $\langle\langle$ weak equivalence class $\rangle\rangle$ contains a strongly measurable mapping is the object of a classical theorem due to R. S. Phillips [9]. The known proofs introduce the associated weakly compact operator of $L_{R}^{1}$ into $X$ (see [1, Chapter 6 (1959), p. 95, Exercise 25], or [4, pp. 91-92]), or the associated vector-valued measure (see [11, pp. 115-118]) and are quite laborious. The separation property, as exhibited in Theorem 5, makes the notion of lifting appear as a natural tool in this type of problem: We obtain a one-line proof of Phillips' theorem. Furthermore the notion of lifting yields a canonical way of constructing a strongly measurable function $g$ in the weak equivalence class of $f$.

\section{BIBLIOGRAPHY}

1. N. Bourbaki, Eléments de mathématique. Intégration, Actualités Sci. Indust., nos. 1175, 1244, 1281, 1306, 1343, Hermann, Paris, 1952-1969. MR 14, 960; 18, 881; 23 \#A2033; 31 \#3539; 43 \#2183.

2. S. D. Chatterji, Un principe de sous-suites dans la théorie des probabilités, Séminaire de Probabilités VI, Univ. de Strasbourg, Springer-Verlag, Berlin and New York, 1972, pp. $72-89$.

3. A. Ionescu Tulcea and C. Ionescu Tulcea, Liftings for abstract-valued functions and separable stochastic processes, $\mathrm{Z}$. Wahrscheinlichkeitstheorie und Verw. Gebiete 13 (1969), 114-118. MR 43 \#2763.

4. - Topics in the theory of lifting, Ergebnisse der Math. und ihrer Grenzgebiete, Band 48, Springer-Verlag, New York, 1969. MR 43 \#2185

5. A. Ionescu Tulcea, On pointwise convergence, compactness and equicontinuity in the lifting topology. I, Z. Wahrscheinlichkeitstheorie und Verw. Gebiete 26 (1973), 197-205.

6. - On pointwise convergence, compactness and equicontinuity. II, Advances in Math. (to appear).

7. J. Komlós, A generalization of a problem of Steinhaus, Acta Math. Acad. Sci. Hungar. 18 (1967), 217-229. MR 35 \#1071.

8. P. A. Meyer, Représentation intégrale des fonctions excessives. Résultats de Mokobodzki, Séminaire de Probabilités V, Univ. de Strasbourg, Springer-Verlag, Berlin and New York, 1971, pp. 196-208.

9. R. S. Phillips, On weakly compact subsets of a Banach space, Amer. J. Math. 65 (1943), 108-136. MR 4, 218.

10. M. Sion, Group-valued outer measures, Actes Congrès Intern. Math., 1970, Tome 2, pp. 589-593.

11. - A theory of semigroup valued measures, Univ. of British Columbia, Lecture Notes, 1971-72.

Current address: Department of Mathematics, Northwestern University, Evanston, Illinois 60201 